\title{
PHYTOGEOGRAPHICAL ANALYSIS OF EUPHORBIA SUBGENUS ESULA (EUPHORBIACEAE)
}

\author{
DMitry V. GeltMAN
}

\begin{abstract}
Euphorbia subg. Esula is one of four major clades within the genus. A geographical analysis of the 466 species in the subgenus is reported here. Every species was assigned to one of 29 geographical elements clustered in ten groups of elements. This geographical analysis showed that the Tethyan group (comprising nine geographical elements) clearly dominates the subgenus and contains 260 species $(55.79 \%$ of the total number of species). The most numerous geographical elements are Irano-Turanian (105 species) and Mediterranean (85). Other significant groups of elements are Boreal (91 species, 19.54\%), East Asian (40 species, 8.58\%), Madrean (26 species, 5.58\%), Paleotropical (23 species, 4.94\%) and South African (16 species, $3.43 \%$ ). The area of the Tethyan floristic subkingdom is the center of the modern diversity of E. subg. Esula. It is likely that such diversity is the result of intensive speciation that took place during the Eocene-Miocene.
\end{abstract}

Key words: Euphorbia subg. Esula, geographical elements, Irano-Turanian floristic region, Mediterranean floristic region, phytogeographical analysis, Tethyan floristic subkingdom

Dmitry V. Geltman, Komarov Botanical Institute of the Russian Academy of Sciences, Prof. Popov Street, 2, St. Petersburg, 197376, Russia; e-mail: geltman@binran.ru

\section{INTRODUCTION}

\section{GENUS EUPHORBIA AND ITS TAXONOMY}

The giant genus Euphorbia L. (Euphorbiaceae) recently became a subject of detailed phylogenetic and taxonomic studies. Four major clades (A-D) were identified in the first phylogenetic investigation of Euphorbia by Steinmann and Porter (2002). These clades were later confirmed and formalized by Bruyns et al. (2006) as four subgenera: Euphorbia subg. Rhizanthium (Boiss.) Wheeler (recently an earlier name, E. subg. Athymalus Neck. ex Rchb., was found for this taxon - Peirson et al. 2013), E. subg. Esula Pers., E. subg. Euphorbia, and E. subg. Chamaesyce Raf. The concept of four clades/subgenera was supported by further studies (Park \& Jansen 2007; Zimmerman et al. 2010; Bruyns et al. 2011; Horn et al. 2012) and is now accepted by the majority of taxonomists dealing with Euphorbia.

For each clade/subgenus, new taxonomic systems based on robustly sampled molecular phylogenetic analyses have been proposed (Yang et al. 2012; Dorsey et al. 2013; Peirson et al. 2013; Riina et al. 2013). In many cases the new systems signifi- cantly differ from traditional ones. For subgenus Esula (Riina et al. 2013), 21 sections were accepted on the basis of analyses of the combined ITS + ndhF dataset (Fig. 1).

The next steps in understanding the evolution and systematics of Euphorbia will involve integrating data from traditional fields of botany (morphology, anatomy, karyology, plant geography, etc.) with the new classification systems for the genus and its subgenera - in other words, to fill the new Euphorbia system with morphological, geographical and other content.

Riina et al. (2013) gave a short biogeographical sketch of the subgenus but their work did not involve a more detailed phytogeographical study. The present paper reports the results of my phytogeographical analysis of the species comprising E. subg. Esula.

\section{CONCEPT OF PHYTOGEOGRAPHICAL ELEMENTS}

The most important part of any phytogeographical analysis consists in assigning every species (of a flora or taxon) to a particular geographical 


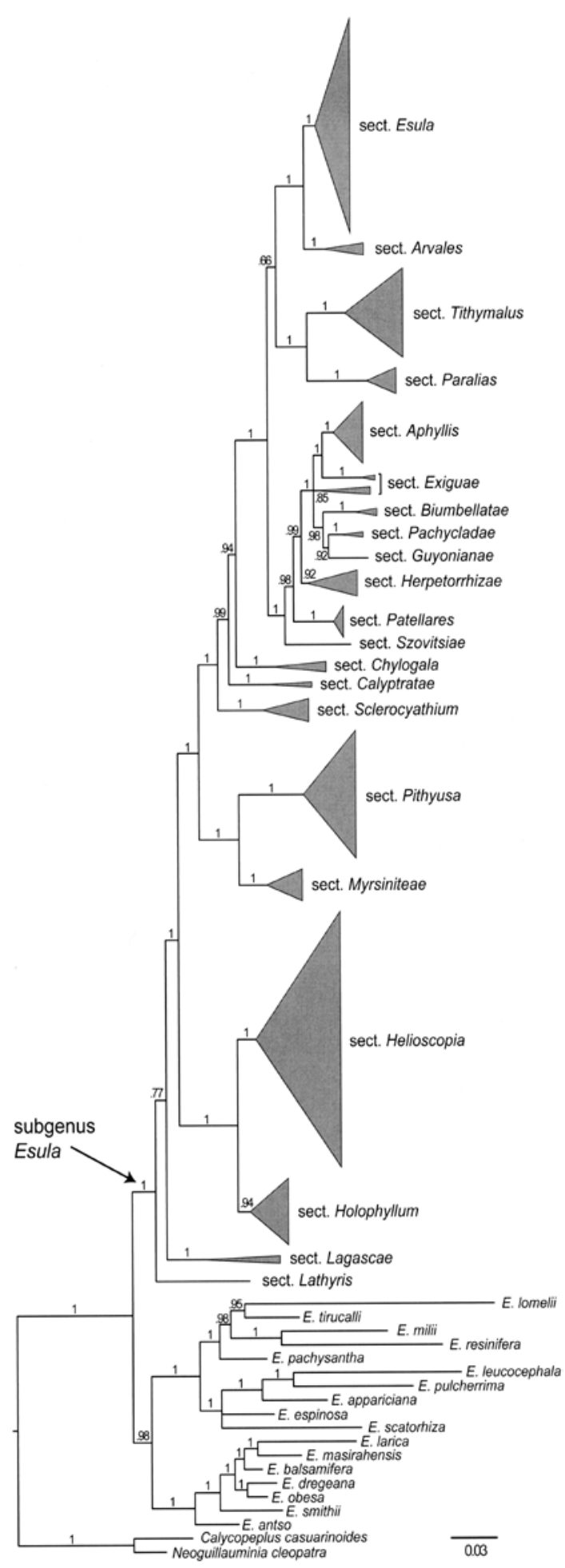

element. Such assignments have been made in a number of floras (e.g., Davis 1965-1988); detailed phytogeographical analyses of particular areas, with quantitative assessments, are the subject of special publications (e.g., Ali \& Qaiser 1986). Such assignments and analyses can also be found in taxonomic reviews (e.g., Skvortsov 1966; Menitsky 1986, 1992; Budantsev 1998; Kurosawa 2006), and this practice is especially common in the Russian school of plant taxonomy and phytogeography.

Generally, a geographical element is a group of species with a similar distribution pattern. Tolmachev (1974: 134) mentioned that 'species ... having more or less similar geographical distributions are regarded as a geographical element of a particular flora, and the element is named according to the details of such a kind of distribution'.

The term 'element' appeared in early works on phytogeography (e.g., Christ 1867; Engler 1879). The modern approach in phytogeographical analysis is connected with works of Braun-Blanquet (1919, 1923) and Eig (1931). Braun-Blanquet (1923: 32) stated that 'l'élément phytogéographique est l'expression floristique et phytosociologique d'un territoire étendu défini; il englobe les 'sippes' et les collectivités phytogéographiques caractéristiques d'une région déterminée'. A similar definition of 'geographical element' was made by Davis (1965): '[a phytogeographical] element ... is a taxon confined or centered in one phytogeographical region (or province). A species whose maximum abundance is clearly in one region is treated as an element of this region. Such an element may occur locally in communities of another region, or in association with other elements of its own region, forming enclaves isolated from the main area'. Such an approach is often called a flo-

Fig. 1. Schematic representation of Bayesian phylogram from analysis of the combined ITS + ndhF dataset of Euphorbia subg. Esula Pers. and outgroups. Sections are represented by filled triangles whose vertical axes are scaled to reflect the number of species in each section. Bayesian posterior probabilities $(\geq 0.66)$ are indicated above the branches (from Riina et al. 2013, fig. 4 - reproduced with permission from the International Association for Plant Taxonomy). 
ristic one in the classification of geographical elements (Portenier 2000a), but Yurtsev and Kamelin (1991: 43) defined it as 'chorionomic' and also distinguished 'coordinate' and 'relative' types of geographical elements.

Here I follow the above mentioned floristic (chorionomic) approach to the classification of geographical elements. Recently this approach was developed by Portenier $(2000 \mathrm{a}, \mathrm{b})$ in connection with work on the flora of the Caucasus. According to his concept, the term 'geographical element' is connected with phytochoria of various ranks (kingdoms, subkingdoms, regions, provinces, etc.). A geographical element is a set of species comprising the nucleus of a flora of a concrete phytochorion, and such species also are members of plant communities determining the specificity of the vegetation of the phytochorion.

As the basic phytochorion for the current analysis I selected the floristic region, although in other cases (depending on the aim and scope of the analysis) other levels of floristic division could be selected (e.g., province or even district).

It is not problematic to assign a species to a geographical element if it occurs within the limits of a single floristic region only. In this case the name of the element usually repeats the name of the phytochorion. The situation is more complicated if a species occurs in two or more floristic regions without evident preferences for one or the other. In this case one can consider 'plantes de liaison' (Eig 1931: 13), binding species (or elements) (Portenier 2000a), or bi- and pluriregional elements (Ali \& Qaiser 1986).

I follow Portenier (2000b) in treating geographical elements as clustered into groups of elements which mainly correspond with the scheme of floristic subkingdoms or kingdoms.

\section{MATERIAL AND METHODS}

The distribution of every species of $E$. subg. Esula was analyzed and compared with the accepted scheme of floristic zoning (see below). For data on the geographical distribution of species, standard floras, the World Checklist of Euphorbiaceae (Govaerts et al. 2015) and especially the 'Euphorbia Planetary Biodiversity Inventory database' (Riina \& Berry 2015) were used. However, in several cases, especially for species occurring in areas of my special interest (Eastern and Central Europe, the Caucasus, Asia Minor, Central Asia, Siberia), original data from herbarium holdings (first of all LE, but also AA, BAK, BM, E, ERE, FRU, K, KW, MHA, MW, P, TASH, TBI, TGM) were very important.

\section{ACCEPTED SCHEME OF FLORISTIC ZONING}

The most developed and generally accepted system of floristic regions, proposed by Takhtajan (1986), was selected as the basis for this work, modified as described below.

1. The East Asian region is separated into SinoJapanese and Sino-Himalayan regions; their limits generally match those of the Sino-Japanese and Sino-Himalayan subkingdoms proposed by $\mathrm{Wu}$ and $\mathrm{Wu}$ (1998), but the Sino-Himalayan region is extended to cover all of the Western Himalayas, as recently proposed by Kamelin (2010).

2. The Crimean-Novorossiysk province is moved from the Mediterranean to the Circumboreal region, as proposed by Portenier (2000a).

3 . The Hyrcanian province is moved from the Irano-Turanian to the Circumboreal province. Although its flora contains several Irano-Turanian elements, it has more links with Caucasian and Euxine provinces, which are traditionally placed in the Circumboreal province.

4. The limit between the Circumboreal and Irano-Turanian regions is changed such that the Altay-Dhungarian and Tuva-Mongolian provinces recently proposed by Kamelin (2010) are placed in the Circumboreal region.

As mentioned above, geographical elements are clustered into groups of elements according to the scheme of floristic subkingdoms (sometimes kingdoms). Takhtajan's (1986) scheme was accepted as the basis for such clustering, but with some important additions recently proposed by Kamelin (2012):

1. The East Asian subkingdom covering the Sino-Japanese and Sino-Himalayan regions is added.

2. A new South African kingdom is added, covering not only the Cape region but also much more extensive areas of South Africa: the Karoo-Namib region, the south part of the Tongoland-Pondolan 
province of the Uzumbara-Zululand region, and the montane part of the Zambesian province of the Sudano-Zambesian region in Takhtajan's system.

\section{SPECIES COMPOSITION}

The species composition and sectional delimitation of Euphorbia subg. Esula are generally accepted according to Riina et al. (2013), with the following amendments.

Euphorbia lathyris L. (the only species of section Lathyris Dumort.) is excluded from the analysis because it is impossible to determine its native area: it is found in the wild in the Mediterranean area, Central Europe and East Asia, but it is unclear which area is primary. It is likely that this species was developed in cultivation.

Euphorbia section Holophyllum (Prokh.) Prokh.: E. fischeriana Steudel is regarded as the priority name for E. pallasii Turcz.

Euphorbia section Helioscopia Dumort.: E. polychroma A. Kern. and E. cybirensis Boiss. are excluded and treated as synonyms of $E$. epithymoides L. and E. valerianifolia Lam. respectively. E. donii Oudejans and E. schilingii Radcl.-Sm. according to Kurosawa (2006) are treated as synonyms of E. pseudosikkimensis (Hurus. \& Yu. Tanaka) Radcl.-Sm., and E. hakutosanensis Hurus. as a synonym of $E$. fauriei H. Lév., following Chang et al. (2014). E. insularis Boiss. and E. canuiti Parl. (commonly treated as subspecies of E. hyberna L.), as well as the recently described E. mazandaranica Pahlevani (Pahlevani \& Riina 2014) and E. maoershanensis F. N. Wei \& J. S. Ma ${ }^{1}$ (Ma et al. 2013), are added. The enigmatic E. peltata Roxb., described in 1832 from the Coromandel coast in India and never collected later, is likely a synonym of the weedy E. stricta L. which has occasionally been introduced from Europe. E. sendaica Makino, after closer examination, was moved to section Esula (Pers.) Dumort.

Euphorbia normannii Schmalh. ex Lipsky was moved from section Arvales (Geltman) Geltman

\footnotetext{
1 The authors of E. maoershanensis Wei \& Ma (Ma et al. 2013) included their species in section Holophyllum. In my opinion, this species more likely belongs to section Helioscopia.
}

to section Myrsiniteae (Boiss.) Lojac., according to newly obtained molecular data (A. Kryukov, D. Geltman, unpubl. data).

Euphorbia section Pithyusa (Raf.) Lázaro: the recently described E. khorasanica Saeidi \& Ghayormand (Saeidi Mehrvarz \& Ghayormand 2015) as well as the previously known E. antilibanotica Mouterde, E. cuspidata Bertol. and E. sanctaecatarinae Fayed, suggested by Riina et al. (2013) as possible members of this section, are added; however, E. sanctae-catarinae could also belong to section Paralias ${ }^{2}$.

Euphorbia section Patellares (Prokh.) Frajman: E. meuselii Geltman (=E. amygdaloides L. subsp. arbuscula Meusel) and E. wulfenii Hoppe ex W. D. J. Koch (=E. characias L. subsp. wulfenii (Hoppe ex W. D. J. Koch) Radcl.-Sm. are added.

The New World species of section Tithymalus (Gaertn.) Roep. are accepted according to a recent revision (Peirson et al. 2014).

Euphorbia section Esula (Pers.) Dumort.: E. discolor Ledeb. is excluded from the analysis, because this name is a later homonym; the information connected with this name should be attributed to E. borealis Baikov (Baikov 2007). Euphorbia lioui C. Y. Wu \& J. A. Ma and E. gulestanica Podlech are added.

Euphorbia ensifolia Baker from Madagascar, close to section Esula according to Riina et al. (2013), is not included in the analysis because I have doubts as to its belonging to E. subg. Esula.

\section{RESULTS}

In total, 466 species were included in the analysis. Following the above proposed concept I have separated 29 geographical elements clustered in ten groups of elements. The results of the analysis are summarized in Tables 1 and 2.

Below are brief descriptions of all elements, with lists of the species assigned to them. Uniregional elements have the same names as those of the floristic regions; it is assumed that the distribu-

\footnotetext{
2 Euphorbia elymaitica Bornm., which was not placed in any particular section by Riina et al. (2013), most probably is a synonym of E. microsciadia Boiss.
} 
tion areas of species belonging to those elements are within the particular region.

\section{BOREAL GROUP (91 species)}

1. West-Palearctic element (pluriregional). Portenier (2000b) assigned to this element species with distributions in Europe, West Siberia and the western part of Tethyan subkingdom, and I follow this definition. Species (2): E. seguieriana Neck., E. virgata Waldst. \& Kit.

2. Euro-Siberian element (uniregional). Species occurring in the Eurasian part of the Circumboreal floristic region, which is often separated as the Euro-Siberian region (Hayek 1926; Good 1964; Davis 1965). Species (81): E. agraria M. Bieb., E. alpina Ledeb., E. altaica Ledeb., E. amygdaloides L., E. angulata Jacq., E. ardonensis Galushko, E. aristata Schmalh., E. austriaca A. Kern., E. borealis Baikov, E. borodinii Sambuk, E. buchtormensis Ledeb., E. buschiana Grossh., E. caesia Kar. \& Kir., E. carniolica Jacq., E. carpatica Wol., E. cyparissias L., E. czerepanovii Geltman, E. daghestanica Geltman, E. djimilensis Boiss., E. dubovikiae Oudejans, E. dulcis L., E. epithymoides L., E. eugeniae Prokh., E. esula L., E. fischeriana Steudel, E. glaberrima K. Koch, E. gmelinii Steud., E. gregersenii K. Malý ex Beck, E. hyberna L., E. iberica Boiss., E. illirica Lam., E. jenisseiensis Baikov, E. kaleniczenkoi Czern., E. kerneri Huter ex A. Kern., E. kirimzjulica Stepanov, E. korshinskyi Geltman, E. latifolia Ledeb., E. lenensis Baikov, E. leptocaula Boiss., E. lucida Waldst. \& Kit., E. macroceras Fisch. \& C. A. Mey., E. macrorhiza Ledeb., E. mazandaranica Pahlevani, E. microcarpa (Prokh.) Krylov, E. mongolica (Prokh.) Prokh., E. normannii Schmalh. ex Lipsky, E. oblongifolia (K. Koch) K. Koch, E. palustris L., E. pancicii Beck, E. pannonica Host, E. pilosa L., E. platyphyllos L., E. polygalifolia Boiss. \& Reut., E. potaninii Prokh., E. procera M. Bieb., E. pseudagraria P. A. Smirn., E. pyrenaica Jord., E. rossica P. A. Smirn., E. rupestris Ledeb., E. salicifolia Host, E. sareptana Becker, E. saurica Baikov, E. saxatilis Jacq., E. scripta Sommier \& Levier, E. semivillosa (Prokh.) Krylov, E. sojakii (Chrtek \& Křísa) Dubovik, E. squamosa Willd.,
E. stepposa Zoz, E. subamplexicaulis Kar. \& Kir., E. subcordata Ledeb., E. subtilis (Prokh.) Prokh., E. tauricola Prokh., E. tristis Besser, E. tshuiensis (Prokh.) Serg. ex Krylov, E. undulata M. Bieb., E. uralensis Fisch. ex Link, E. valdevillosocarpa Arvat \& Nyár., E. valliniana Belli, E. variabilis Ces., E. verrucosa L., E. wittmannii Boiss.

3. North American Atlantic element (uniregional). Species (5): E. georgiana M. H. Mayfield, E. longicruris Scheele, E. ouachitana M. H. Mayfield, E. purpurea (Raf.) Fernald, E. texana Boiss.

4. North American element (pluriregional). Species found in at least in two of the following regions: Circumboreal (American part), North American Atlantic, Rocky Mountain and Madrean. Species (3): E. brachycera Engelm., E. commutata Engelm. ex A. Gray, E. spathulata Lam.

\section{EAST ASIAN GROUP (40 species)}

5. Sino-Japanese element (uniregional). The Sino-Japanese floristic region is accepted here within the limits of the following provinces according to Takhtajan (1986): Manchurian, Sakhalin-Hokkaido, Japanese-Korean, VolcanoBonin, Ryukyu (Tokara-Okinawa), Taiwanian, Northern Chinese and Central Chinese (Wu \& Wu 1998). Species (20): E. adenochlora C. Morren \& Decne., E. dahurica Peschkova, E. ebracteolata Hayata, E. fauriei H. Lév. \& Vaniot, E. jolkinii Boiss., E. kansuensis Prokh., E. kansui S. L. Liou, E. komaroviana Prokh., E. lucorum Rupr., E. lunulata Bunge, E. mandshurica Maxim., E. maoershanensis F. N. Wei \& J. S. Ma, E. nakaii Hurus., E. octoradiata H. Lév. \& Vaniot, E. pekinensis Rupr., E. sendaica Makino, E. sieboldiana C. Morren \& Decne., E. togakusensis Hayata, E. tongchuanensis C. Y. Wu \& J. S. Ma, E. yanjinensis W. T. Wang.

6. Sino-Himalayan element (uniregional). The Sino-Himalayan floristic region is accepted here within the limits of the following provinces according to Takhtajan (1986): Southeastern Chinese, Sikang-Yunnan, Northern Burmese, Eastern Himalayan, Khasi-Manipur (Wu \& Wu 1998), plus the Western Himalayan province (following Kamelin 2010) which Takhtajan placed in the 
Table 1. Distribution of species of Euphorbia subg. Esula among geographical elements and groups of elements.

\begin{tabular}{|c|c|c|c|c|c|c|}
\hline $\begin{array}{l}\text { Group of } \\
\text { elements }\end{array}$ & $\begin{array}{l}\text { Number of } \\
\text { species in } \\
\text { the group }\end{array}$ & $\begin{array}{c}\% \text { of total } \\
\text { species } \\
\text { number }\end{array}$ & Element & $\begin{array}{l}\text { Number } \\
\text { of species }\end{array}$ & $\begin{array}{c}\% \text { of total } \\
\text { number }\end{array}$ & $\begin{array}{c}\% \text { of number of } \\
\text { species in group } \\
\text { of elements }\end{array}$ \\
\hline \multirow[t]{4}{*}{ BOREAL } & \multirow[t]{4}{*}{91} & \multirow[t]{4}{*}{19.54} & West-Palearctic & 2 & 0.43 & 2.20 \\
\hline & & & \begin{tabular}{|l|} 
Euro-Siberian \\
\end{tabular} & 81 & 17.38 & 89.01 \\
\hline & & & North American Atlantic & 5 & 1.07 & 5.49 \\
\hline & & & North American & 3 & 0.64 & 3.30 \\
\hline \multirow[t]{2}{*}{ EAST ASIAN } & \multirow[t]{2}{*}{40} & \multirow[t]{2}{*}{8.58} & Sino-Japanese & 20 & 4.29 & 50 \\
\hline & & & Sino-Himalayan & 20 & 4.29 & 50 \\
\hline \multirow{9}{*}{$\begin{array}{l}\text { TETHYAN } \\
\text { (ANCIENT } \\
\text { MEDITERRANEAN) }\end{array}$} & \multirow[t]{9}{*}{260} & \multirow[t]{9}{*}{55.79} & Pantethyan & 2 & 0.43 & 0.77 \\
\hline & & & European-Pantethyan & 8 & 1.72 & 3.08 \\
\hline & & & Macaronesian & 14 & 3.00 & 5.39 \\
\hline & & & Mediterranean & 85 & 18.24 & 32.69 \\
\hline & & & Macaronesian-Mediterranean & 7 & 1.50 & 2.69 \\
\hline & & & Submediterranean & 32 & 6.87 & 12.31 \\
\hline & & & Saharo-Arabian & 4 & 0.86 & 1.54 \\
\hline & & & Irano-Turanian & 105 & 22.53 & 40.38 \\
\hline & & & Irano-Turanian-Sino-Himalayan & 3 & 0.64 & 1.15 \\
\hline \multirow[t]{2}{*}{ MADREAN } & \multirow[t]{2}{*}{26} & \multirow[t]{2}{*}{5.58} & Madrean & 18 & 3.86 & 69.23 \\
\hline & & & Submadrean & 8 & 1.72 & 30.77 \\
\hline \multirow[t]{2}{*}{ PALEOTROPICAL } & \multirow[t]{2}{*}{23} & \multirow[t]{2}{*}{4.94} & Panpaleotropical & 1 & 0.21 & 4.35 \\
\hline & & & Sudano-Zambesian & 22 & 4.72 & 95.65 \\
\hline MADAGASCAN & 3 & 0.64 & Madagascan & 3 & 0.64 & 100 \\
\hline \multirow[t]{2}{*}{ INDOMALESIAN } & \multirow[t]{2}{*}{2} & \multirow[t]{2}{*}{0.43} & Indomalesian & 1 & 0.22 & 50 \\
\hline & & & Fijian & 1 & 0.22 & 50 \\
\hline NEOTROPICAL & 3 & 0.64 & Carribean & 3 & 0.64 & 100 \\
\hline \multirow[t]{4}{*}{ SOUTH AFriCAN } & \multirow[t]{4}{*}{16} & \multirow[t]{4}{*}{3.43} & Karoo-Namib & 1 & 0.22 & 6.25 \\
\hline & & & Uzambara-Zululand & 4 & 0.86 & 25.00 \\
\hline & & & Cape & 4 & 0.86 & 25.00 \\
\hline & & & South African & 7 & 1.50 & 43.75 \\
\hline \multirow[t]{2}{*}{ GOLANTARCTIC } & \multirow[t]{2}{*}{2} & \multirow[t]{2}{*}{0.43} & Chile-Patagonian & 1 & 0.22 & 50 \\
\hline & & & Neozeylandic & 1 & 0.22 & 50 \\
\hline
\end{tabular}

Irano-Turanian region. The Sino-Himalayan species can also be found in exclaves in Tibet. Species (20): E. cashmeriana Royle, E. cornigera Boiss., E. griffithii Hook. f., E. heishuiensis W. T. Wang, E. himalayensis (Klotzsch) Boiss., E. hylonoma Hand.-Mazz., E. jacquemontii Boiss., E. khasyana Boiss., E. kingdonwardii Binojkumar \& N. P. Balakr., E. luteoviridis D. G. Long, E. maddenii Boiss., E. micractina Boiss., E. prolifera Buch.Ham. ex D. Don, E. pseudosikkimensis (Hurus. \& Yu. Tanaka) Radcl.-Sm., E. saxicola Radcl.-Sm., E. sharmae Battacharyya, E. sikkimensis Boiss., E. stracheyi Boiss., E. thyrsoidea Boiss., E. wallichii Hook. f.

\section{Tethyan (ANCIENT MediterraneAn) GrouP (260 species)}

7. Pantethyan element (pluriregional). Plants found in at least three regions of the Tethyan (Ancient Mediterranean) floristic subkingdom; sometimes they have a wider secondary distribution. Species (2): E. chamaepeplus Boiss. \& Gaill., E. dracunculoides Lam.

8. European-Pantethyan element (pluriregional). Usually widely distributed species occurring in at least two regions of the Tethyan floristic subkingdom and also in the European part of the Circumboreal region, at least in the Atlantic and 


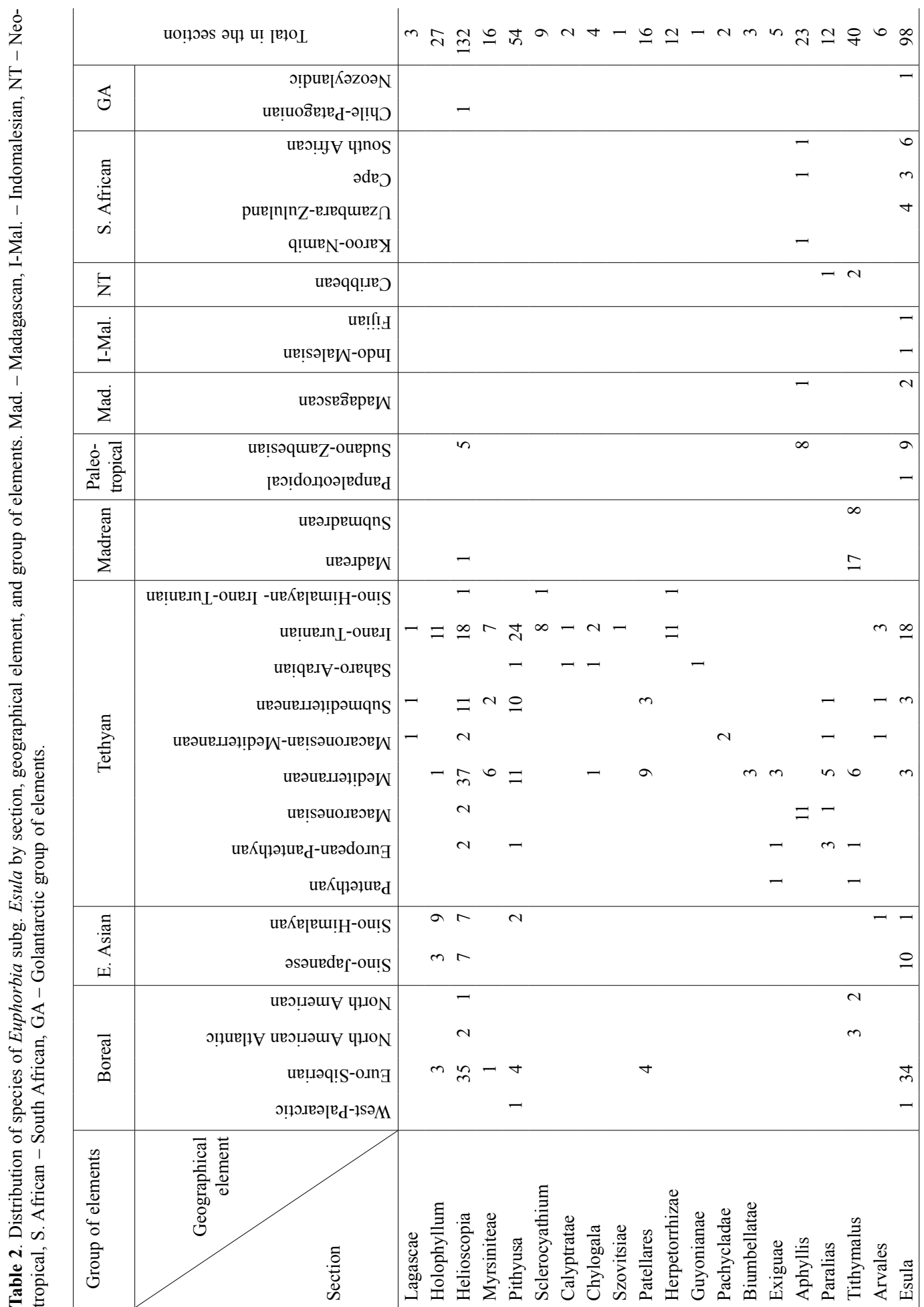


Central European provinces. Most of the species of this group are weeds, and it is impossible to separate their primary and secondary distribution areas. Species (8): E. exigua L., E. falcata L., E. helioscopia L., E. paralias L., E. peplus L., E. portlandica L., E. stricta L., E. taurinensis All.

9. Macaronesian element (uniregional). Species (14): E. anachoreta Svent., E. aphylla Brouss. ex Willd., E. atropurpurea Brouss., E. azorica Hochst., E. berthelotii Bolle ex Boiss., E. bourgaeana J. Gay ex Boiss., E. bravoana Svent., E. lamarckii Sweet, E. mellifera Aiton, E. pedroi Molero \& Rovira, E. piscatoria Aiton, E. regisjubae Webb \& Berthel., E. stygiana H. C. Watson, E. tuckeyana Steud. ex Webb.

10. Mediterranean element (uniregional). Species (85): E. acanthothamnos Heldr. \& Sart. ex Boiss., E. akenocarpa Guss., E. aleppica L., E. antilibanotica Mouterde, E. anthula Lavrent. \& Papan., E. apios L., E. arguta Banks \& Sol., E. austroanatolica Hub.-Mor. \& M. S. Khan, E. barrelieri Savi, E. berythea Boiss. \& Blanche, E. biumbellata Poir., E. bivonae Steud., E. boetica Boiss., E. briquetii Emb. \& Maire, E. bupleuroides Desf., E. canuti Parl., E. capitulata Rchb., E. caudiculosa Boiss., E. celerieri (Emb.) Emb. ex Vindt, E. ceratocarpa Ten., E. characias L., E. clementei Boiss., E. corallioides L., E. corsica Req., E. cossoniana Boiss., E. cuneifolia Guss., E. davisii M. S. Khan, E. deflexa Sibth. \& Sm., E. dimorphocaulon P. H. Davis, E. durandoi Chabert, E. erinacea Boiss. \& Kotschy, E. fontqueriana Greuter, E. gaditana Coss., E. gasparrinii Boiss., E. gayi Salis, E. gebelica Brullo, E. graminifolia Vill., E. haussknechtii Boiss., E. herniariifolia Willd., E. hieroglyphica Coss. \& Durieu ex Boiss., E. hierosolymitana Boiss., E. insularis Boiss., E. isatidifolia Lam., E. isaurica M. S. Khan, E. kotschyana Fenzl, E. lagascae Spreng., E. maresii Knoche, E. margalidiana Kuhbier \& Lewej., E. matritensis Boiss., E. mazicum Emb. \& Maire, E. medicaginea Boiss., E. megalatlantica Ball, E. melapetala Gasp. ex Guss., E. melitensis Parl., E. meuselii Geltman, E. minuta Loscos \& Pardo, E. nereidum Jahand. \& Maire, E. nevadensis Boiss. \& Reut., E. nicaeensis All., E. nurae P. Fraga \& Rossello, E. ob- longata Griseb., E. oxyphylla Boiss., E. paniculata Desf., E. papillaris (Boiss.) Raffaelli \& Ricceri, E. pestalozzae Boiss., E. pisidica Hub.-Mor. \& M. S. Khan, E. pithyusa L., E. promecocarpa Davis, E. pseudoapios Maire \& Weiller, E. punctata Delile, E. rechingeri Greuter, E. reuteriana Boiss., E. rhytidosperma Boiss. \& Balansa, E. rimarum Coss. \& Balansa, E. schottiana Boiss., E. semiperfoliata Viv., E. serrata L., E. sintenisii Boiss. ex Freyn, E. spinosa L., E. squamigera Loisel., E. sultan-hassei Strid et al., E. thompsonii Holmboe, E. transtagana Boiss., E. valerianifolia Lam., E. veneris M. L. S. Khan., E. wulfenii Hoppe ex W. D. J. Koch.

11. Macaronesian-Mediterranean element (biregional). Plants occurring in both the Mediterranean (usually western) and Macaronesian regions. It is quite possible that at least some of these species originally occurred in the Mediterranean only and then were introduced to Macaronesia, but it is now impossible to present indisputable evidence of this. Species (7): E. dendroides L., E. hirsuta L., E. lagascae Spreng., E. pterococca Brot., E. segetalis L., E. sulcata Lens ex Loisel, E. terracina L.

12. Submediterranean element (uni- or pluriregional). This element incorporates species having different distribution patterns but in some way connected with the Mediterranean region. Within this element at least three principal groups can be separated: (a) species occurring in the Mediterranean region itself (usually at higher elevations) and also in neighboring areas like the Balkans, Caucasus, Crimea or Anatolia (E. myrsinites, E. flavicoma, E. rigida, etc.); (b) species found in the Mediterranean and/or at the western edge of the IranoTuranian region and at the same time in southern parts of the Circumboreal region, where such plants are usually restricted to 'Mediterraneanlike' habitats such as exposed rocks or limestone outcrops (E. condylocarpa, E. glareosa, E. petrophila); (c) species endemic to mountain systems located at the northern border of the Mediterranean, or local endemics to areas adjoining the Mediterranean (Crimean-Novorossiysk, Euxine, Illirian provinces) and occupying 'Mediterraneanlike' habitats (E. heldreichii, E. orphanidis, E. panjutinii). Species (32): E. androsaemifolia Willd. 
ex Schlecht., E. aulacosperma Boiss., E. condylocarpa M. Bieb., E. duvalii Lecoq \& Lamotte, E. erubescens Boiss., E. erythrodon Boiss. \& Heldr., E. filicina Port., E. flavicoma DC., E. fragifera Jan, E. glabriflora Vis., E. glareosa Pall. ex M. Bieb., E. heldreichii Orph. ex Boiss., E. hercegovina Beck, E. imperfoliata Vis., E. ledebourii Boiss., E. microsphaera Boiss., E. montenegrina (Bald.) K. Malý, E. myrsinites L., E. niciciana Borbás ex Novák, E. obovata Decne., E. orjeni Beck, E. orphanidis Boiss., E. panjutinii Grossh., E. petrophila C. A. Mey., E. phymatosperma Boiss. \& Gaill., E. rhabdotosperma Radcl.-Sm., E. rigida M. Bieb., E. serpentini Novák, E. thessala (Formánek) Degen \& Dörfl., E. triflora Schott et al., E. uliginosa Welw. ex Boiss., E. velenovskyi Bornm.

13. Saharo-Arabian element (uniregional). Species (4): E. calyptrata Coss. \& Durieu, E. guyoniana Boiss. \& Reut., E. retusa Forssk., E. sanctae-catarinae Fayed.

14. Irano-Turanian element (uniregional). As mentioned above, in the current analysis the Western Himalayan province is excluded here from the Irano-Turanian region. Species (105): E. acanthodes Akhani, E. alaica (Prokh.) Prokh., E. alatavica Boiss., E. altissima Boiss., E. altotibetica Paulsen, E. anacampseros Boiss., E. andrachnoides Schrenk, E. armena Prokh., E. arvalis Boiss. \& Heldr., E. aserbajdzhanica Bordz., E. astrachanica C. A. Mey. ex Trautv., E. aucheri Boiss., E. blatteri Oudejans, E. blepharophylla Ledeb., E. buhsei Boiss., E. bungei Boiss., E. caeladenia Boiss., E. cassia Boiss., E. chaborasia Gomb., E. cheiradenia Boiss. \& Hohen., E. coniosperma Boiss. \& Buhse, E. connata Boiss., E. consanguinea Schrenk, E. craspedia Boiss., E. cuspidata Bertol., E. cyrtophylla (Prokh.) Prokh., E. deltobracteata (Prokh.) Prokh., E. densa Schrenk, E. densiuscula M. Pop., E. densiusculiformis (Pazij) Botsch., E. denticulata Lam., E. eriophora Boiss., E. erythradenia Boiss., E. ferganensis B. Fedtsch, E. fistulosa M. S. Khan, E. franchetii B. Fedtsch., E. gaillardotii Boiss. \& Blanche, E. gedrosiaca Rech. f. et al., E. glomerulans (Prokh.) Prokh., E. grisophylla M. S. Khan, E. grossheimii (Prokh.) Prokh., E. guntensis (Prokh.) Prokh., E. gypsi- cola Rech. f. \& Aellen, E. gulestanica Podlech, E. hebecarpa Boiss., E. heptapotamica Golosk., E. heteradena Jaub. \& Spach, E. humilis Ledeb., E. hyrcana Grossh., E. inderiensis Less. ex Kar. \& Kir., E. iranshahri Pahlevani, E. irgisensis Litv., E. jaxartica (Prokh.) Krylov, E. khorasanica Saeidi \& Ghayormand, E. kopetdaghi (Prokh.) Prokh., E. kozlovii Prokh., E. kudrjaschevii (Pazij) Prokh., E. lamprocarpa (Prokh.) Prokh., E. lioui C. Y. Wu \& J. S. Ma, E. lipskyi (Prokh.) Prokh., E. macrocarpa Boiss. \& Buhse, E. macroclada Boiss., E. malleata Boiss., E. malurensis Rech. f., E. marschalliana Boiss., E. megalocarpa Rech. f., E. microsciadia Boiss., E. monocyathium (Prokh.) Prokh., E. monostyla Prokh., E. mucronulata (Prokh.) Pavlov, E. oidorrhiza Pojark., E. orientalis L., E. osyridea Boiss., E. oxyodonta Boiss., E. pachyrrhiza Kar. \& Kir., E. pamirica (Prokh.) Prokh., E. pauciradiata Blatt., E. physocaulos Mouterde, E. plebeia Boiss., E. poecilophylla (Prokh.) Prokh., E. polycaula Boiss. \& Hohen., E. rapulum Kar. \& Kir., E. rosularis Fed., E. sahendii Bornm., E. sarawschanica Regel, E. schugnanica B. Fedtsch., E. sclerocyathium Korovin \& Popov, E. sewerzowii (Prokh.) Pavlov, E. smirnovii Geltman, E. sogdiana Popov, E. soongarica Boiss., E. sororia Schrenk, E. spartiformis Mobayen, E. spinidens Bornm. ex Prokh., E. szovitsii Fisch. \& C. A. Mey., E. talaina Radcl.-Sm., E. talastavica (Prokh.) Prokh., E. teheranica Boiss., E. thomsoniana Boiss., E. tianshanica (Prokh.) Popov, E. transoxana (Prokh.) Prokh., E. triodonta (Prokh.) Prokh., E. turczaninowii Kar. \& Kir., E. turkestanica Regel, E. yaroslavii Poljakov.

15. Sino-Himalayan-Irano-Turanian element (biregional). Species occurring in montane areas of the Irano-Turanian region (Pamir, Tien-Shan, Tibet) and also in the Sino-Himalayan region (mainly in the Himalayas). Species (3): E. edgeworthii Boiss., E. kanaorica Boiss., E. tibetica Boiss.

\section{MADREAN GROUP (26 species)}

16. Madrean element (uniregional). Species (18): E. alta Norton, E. beamanii M. C. Johnst., E. chamaesula Boiss., E. chiribensis V. W. Steinm. \& Felger, E. correllii M. C. Johnst., E. creberrima 
McVaugh, E. crenulata Engelm., E. cressoides M. C. Johnst., E. greggii Engelm. ex Boiss., E. ivanjohnstonii M. C. Johnst., E. longicornuta S. Watson, E. lurida Engelm., E. mcvaughiana M. C. Johnst., E. neilmuelleri M. C. Johnst., E. nesomii M. H. Mayfield, E. pinkavana M. C. Johnst., E. schizoloba Engelm., E. yaquiana (Cockerell) Tidestr.

17. Submadrean element (biregional). Species found in the Madrean region and also in neighboring areas or in the transitional zone between Madrean and other floristic regions. Species (8): E. austrotexana M. H. Mayfield, E. esuliformis S. Schauer ex Nees \& S. Schauer, E. furcillata Kunth, E. helleri Millsp., E. orizabae Boiss., E. peplidion Engelm., E. roemeriana Scheele, E. tetrapora Engelm.

\section{Paleotropical group (23 species)}

18. Panpaleotropical element (pluriregional). Plants with a wide distribution at least in the Guineo-Congolian and Sudano-Zambesian regions. Species (1): E. schimperiana Scheele.

19. Sudano-Zambesian element (uniregional). Species (22): E. brevicornu Pax, E. calamiformis P. R. O. Bally \& S. Carter, E. citrina S. Carter, E. crebrifolia S. Carter, E. cyparissioides Pax, E. daviesii E. A. Bruce, E. depauperata Hochst. ex A. Rich., E. dumalis S. Carter, E. furcatifolia M. G. Gilbert, E. gossypina Pax, E. lateriflora Schumach., E. nubica N. E. Br., E. pachyclada S. Carter, E. papilionum S. Carter, E. petitiana A. Rich., E. repetita Hochst. ex A. Rich., E. sareciana M. G. Gilbert, E. schimperi C. Presl, E. ugandensis Pax \& K. Hoffm., E. usambarica Pax, E. wellbyi N. E. Br., E. whyteana Baker f.

\section{MADAGASCAN GROUP (3 species)}

20. Madagascan element (uniregional). Species (3): E. borbonica Boiss., E. emirnensis Baker, E. orthoclada Baker.

\section{INDO-MALESIAN GROUP (2 species)}

21. Indo-Malesian element (pluriregional). Plants occurring in the Indian and Malesian re- gions and penetrating to the Indo-China region. Species (1): E. rothiana Spreng.

22. Fijian element (uniregional). Species (1): E. reineckei Pax.

\section{NeOtropicAl GROUP (3 species)}

23. Caribbean element (uniregional). Species (3): E. eggersii Urb., E. trichotoma Kunth, E. tuerckheimii Urb.

\section{SOUth African group (16 species)}

24. Karoo-Namib element (uniregional). Species (1): E. berotica N. E. Br.

25. Uzambara-Zululand element (uniregional). Species (4): E. albanica N. E. Br., E. muraltioides N. E. Br., E. ruscifolia (Boiss.) N. E. Br., E. sclerophylla Boiss.

26. Cape element (uniregional). Species (4): E. erythrina Link, E. foliosa N. E. Br., E. ovata (E. Mey. ex Klotzsch \& Garcke) Boiss., E. stolonifera Marloth.

27. South African element (pluriregional). Plants occurring in at least two of the following regions: Cape, Karoo-Namib, Uzambara-Zululand, and sometimes in neighboring parts of the Sudano-Zambesian region. Species (7): E. epicyparissias (E. Mey. ex Klotzsch \& Garcke) Boiss., E. ericoides Lam., E. genistoides P. J. Bergius, E. kraussiana Bernh. ex Krauss, E. mauritanica L., E. natalensis Bernh. ex Krauss, E. striata Thunb.

\section{Golantarctic group (2 species)}

28. Chile-Patagonian element (uniregional). Species (1): E. philippiana (Klotzsch \& Garcke) Boiss.

29. Neozeylandic element (uniregional). Species (1): E. glauca G. Forst.

\section{Discussion}

DISTRIBUTION OF EUPHORBIA SUBG. ESULA AMONG GEOGRAPHICAL ELEMENTS AND GROUPS OF ELEMENTS

Our data show that species belonging to the TETHYAN GROUP of elements clearly dominate 
Euphorbia subg. Esula (260 species, 55.79\% of the total number). Irano-Turanian (105) and Mediterranean (85) elements are the most numerous, together comprising $73.07 \%$ of the total number of species in the Tethyan group and $40.77 \%$ of the number of species analyzed here.

Species of the Tethyan group are found in all 20 analyzed sections of the subgenus. Members of ten sections [Lagascae Lázaro, Sclerocyatium (Prokh.) Prokh., Calyptratae Geltman, Chylogala (Fourr.) Prokh., Szovitsiae Geltman, Herpetorrhizae (Prokh.) Prokh., Guyonianae Molero \& Riina, Pachycladae (Boiss.) Tutin, Biumbellatae Molero \& Riina, Exiguae (Geltman) Riina $\&$ Molero] belong entirely to the Tethyan group, and three other sections (Patellares, Paralias Dumort., Arvales) have only single or a few species outside that group. Only sections Holophyllum, Aphyllis Webb \& Berthel., Tithymalus and Esula have less than $50 \%$ Tethyan species. Irano-Turanian species are present in 12 sections, and Mediterranean species in 11 sections.

The BOREAL GROUP of elements is represented by 91 species $(19.54 \%)$ and is second in terms of species number. Species of the Euro-Siberian element clearly dominate this group $(89.01 \%$ of total number of species in the group). It needs to be pointed out that most species of the Euro-Siberian element are restricted to western or southern parts of temperate Eurasia (Atlantic-European, Central European, Illyrian, Euxine, Caucasian provinces) and also to the Altay-Sayan and Altay-Dzhungar provinces. If we were to use another scheme of floristic subkingdoms for the analysis, such as that of Kamelin (2010) who included the Caucasus and Balkans into the Tethyan floristic subkingdom, about a quarter of the Euro-Siberian species (first of all endemics and subendemics to the Caucasus and Balkans) could be assigned to the Tethyan group. Species of the Boreal group belong to only seven sections and do not dominate any of them.

Species of the Boreal group occur in both the Old World (Euro-Siberian and West-Palearctic elements, 83 species) and the New World (North American Atlantic and North American elements, 8 species). The latter belong to two sections only (Helioscopia and Tithymalus).
The EAST AsIan group of elements is third in terms of number of species $(40,8.58 \%)$. Those species are equally divided between two elements, but there are no species with a wide distribution in the whole East Asian subkingdom. East Asian species are found in five sections, but mostly in sections Holophyllum, Helioscopia and Esula. Two species of section Pithyusa and one of section Arvales classified here as belonging to the Sino-Himalayan element could in fact occur in exclaves of the Tethyan flora in the Himalayas; this remains to be clarified.

Tethyan, East Asian and Eurasian representatives of the Boreal groups belong to 13 elements and comprise 383 species or $82.19 \%$ of the total number of species in the subgenus. It is clear that the territory of the Tethyan, Boreal (Old World part) and East Asian floristic subkingdoms (in fact, the whole of temperate Eurasia and North Africa) is the main area of the modern diversity of subgenus Esula.

Other groups of elements contain many fewer species but should be thoroughly analyzed because these data can provide very important phylogenetic and phytogeographic information.

There are 38 species of E. subg. Esula native to the New World. Species belonging to the MADREAN GROUP of elements (26 species, 5.58\% of the total number) clearly dominate them, so the Madrean floristic region could be regarded as the center of the modern diversity of E. subg. Esula in the Americas. Eighteen species occur strictly within the limits of the Madrean region, and the other 8 (Submadrean element) extend into neighboring areas. Species of the Madrean group are represented mainly by section Tithymalus (25 species); the only exception is E. alta from section Helioscopia.

Besides species of the Madrean group and the above-mentioned 8 species of the Boreal group in the New World, there are also 3 species of the CARIBBEAN GROUP (with a single Caribbean element) and the enigmatic E. philippiana - a single species of the subgenus native to South America (described from central Chile). Two Caribbean species belong to section Tithymalus and one (E. trichotoma) to section Paralias. 
The Sudano-Zambesian element (22 species) clearly dominates the PALEOTROPICAL GROUP (23 species). Species of this group belong to sections Helioscopia, Aphyllis and Esula. The MADAGASCAN GROUP, with a single element, contains only 3 species: 2 of them (E. emirnensis, E. orthoclada) occur in Madagascar itself, and one is endemic to the island of Réunion, belonging to the Madagascar floristic region. The SoUTH AFRICAN GROUP contains 16 species, and the pluriregional South African element (species with a comparatively wide distribution) is the largest in this group. Only 4 species belong to the Cape element; they are restricted to the Cape province/region, known for its high endemism. Species assigned to the South African and Madagascan groups belong to sections Aphyllis and Esula.

The INDO-MALESIAN GROUP is represented by E. rothiana, with a comparatively wide distribution (Indo-Malesian element), and E. reineckei, endemic to Samoa (Fijian element); both are members of section Esula.

The Golantarctic GROUP is represented by 2 species: E. philippiana (Chile-Patagonian element), mentioned above, and E. glauca (Neozeylandic element).

\section{GEOGRAPHICAL ANALYSIS OF LARGEST SECTIONS}

The members of section Helioscopia, the largest section in the subgenus (132 species), belong to 15 elements clustered in seven groups. As in the whole subgenus, the Tethyan group (73 species, $56.06 \%$ ) predominates. However, Mediterranean species (37) are more than twice as numerous as Irano-Turanian species (18); this may reflect the more mesic character of habitats preferred by species of this section (Riina et al., 2013). A significant number of species $(35$, or $26.52 \%$ of the total number) belong to the Euro-Siberian element. Like the majority of Euro-Siberian species of the whole subgenus, members of section Helioscopia inhabit the Atlantic-European, Central European, Illyrian, Euxine and Caucasian provinces, but 7 species are restricted to the Altay-Sayan and AltayDzhungar provinces. Two species of this section are also found in the New World, and 5 are found in tropical Africa. The section is absent from South Africa and Madagascar, however.

Ninety-eight species of section Esula, the second highest number of species in the subgenus, belong to 16 elements clustered into eight groups. More than a third of its species (34 species, $34.69 \%$ ) belong to the Euro-Siberian element. Species belonging to this section (e.g., E. borodinii, E. gmelinii, E. korshynskyi, E. lenensis) have the northernmost distribution (reaching to the Arctic Circle) of all species of the genus Euphorbia. Ten species of this section belong to the East Asian group, mostly to the Sino-Japanese element. Several Euro-Siberian taxa of section Esula probably are in the process of intensive modern speciation, so species delimitation is uncertain and disputable in several taxonomic groups, for example the E. esula L. s.1. complex (Geltman 1996, 1998) or E. illirica Lam. and closely related species (Geltman 2009).

In section Esula there are 24 species in the Tethyan group (24.49\%). Most of them belong to the Irano-Turanian element and there are only 3 Mediterranean species. Twenty-five species of section Esula occur in tropical Africa, South Africa and Madagascar (Paleotropical, South African and Madagascan groups of elements). There are also 2 species of the Indo-Malesian group (one of them endemic to Samoa) and one endemic to New Zealand. All these species form two well-supported clades which lie outside the temperate Eurasian species (Riina et al. 2013).

Section Pithyusa contains 54 species that belong mainly to the Tethyan group of elements (47 species, 87.04\%), and the number of IranoTuranian species is more than twice that of the Mediterranean species. This section is well known for having the highest proportion of Submediterranean species (10 species, $18.52 \%$ ).

Members of the mostly New World section Tithymalus (40 species) are shared between Madrean (25 species, 62.5\%), Tethyan (8, 20\%) and Boreal (5, all native in the New World, 12.5\%) groups.

Section Holophyllum, with 27 species, has a good representation in the East Asian subkingdom (66.97\%) and there are also 3 Euro- 
Siberian species restricted to southern Siberia and northern Mongolia. Other representatives of this section belong to the Tethyan group, mainly to the Irano-Turanian element, but one species (E. isatidifolia) occurs in the Iberian Peninsula and shows a considerable disjunction from the rest of the section.

Twenty-three species of section Aphyllis are separated into two subsections (Riina et al. 2013); this separation is based on several molecular trees, morphological differentiation, and geographical distribution patterns (Riina et al. 2013; Barres et al. 2011). Subsection Macaronesicae (11 species) has an exclusively Macaronesian distribution. Species of $E$. subsect. Africanae (12 species) occur in Africa and Madagascar and belong mainly to the Sudano-Zambesian element (7 species).

Other sections are comparatively small (less than 15 species, sometimes 1-3) and, as mentioned above, have mainly a Tethyan distribution.

\section{GEOGRAPHICAL ANALYSIS AND PHYLOGENY}

\section{OF THE SUBGENUS}

Data presented here clearly show that the Tethyan floristic subkingdom and adjacent areas are the center of the modern diversity of E. subg. Esula. Very likely, such diversity is the result of intensive speciation that took place here during the EoceneMiocene. Horn et al. (2014) estimated the age of the crown clade of Euphorbia at ca $47.8 \mathrm{Ma}$ and also showed that the divergence of the main lineages within E. subg. Esula took place between ca 38 and $10 \mathrm{Ma}$. This speciation probably proceeded under the influence of two processes: shrinkage of Paratethys (with the formation of desert areas) and Alpine orogenesis. There are some grounds for speculating that orogeny was more important in this, because there are few true desert species of the subgenus.

Only after the formation of the main clades (taxonomically separated as sections) did some species representing such groups migrate to areas outside the original center of diversity. Riina et al. (2013) suggested that there were four independent migrations of the subgenus to the New World, and at least three into tropical and South Africa. The
New World species of section Tithymalus are likely the result of intensive speciation after a single introduction from the Mediterranean area.

It is likely that the colonization of boreal areas of Eurasia took place comparatively recently (although a special phylogeographical study in this area would be desirable) and that it involved representatives of only a few sections, mostly Esula, Helioscopia, and Holophyllum. It seems strange that there are no native species of section Esula in temperate areas of the New World and that they did not use the Beringian land bridge although some of them (e.g., E. lenensis) are found in Siberia near the Arctic circle.

Long-distance dispersal seems the best explanation for the isolated occurrence of some species. The presence of E. glauca in New Zealand and $E$. reineckii on Fiji, at least, is impossible to explain by any schemes of land bridges and continental movements.

ACKNOWLEDGEMENTS. I am grateful to Rudolf Kamelin for numerous valuable consultations, Paul Berry for linguistic corrections of the submitted text and for other comments, and Hans-Joachim (Hajo) Esser and the anonymous reviewer for important suggestions which improved the manuscript. The work was supported by the Russian Foundation for Basic Research (project 13-04-00541). I am grateful to the International Association for Plant Taxonomy for permission to reproduce Figure 1.

\section{REFERENCES}

ALI S. I. \& QAiSER M. 1986. A phytogeographical analysis of the phanerogams of Pakistan and Kashmir. Proc. Roy. Soc. Edinburgh, B 89: 89-101.

BAIKov K. S. 2007. Euphorbia Asiae Borealis. Nauka, Novosobirsk (in Russian).

Barres L., Vilatersana R., Molero J., Susanna A. \& GalBANY-CASALS M. 2011. Molecular phylogeny of Euphorbia subg. Esula sect. Aphyllis (Euphorbiaceae) inferred from nrDNA and cpDNA markers with biogeographic insights. Taxon 60(3): 705-720.

Braun-Blanquet J. 1919. Essai sur les notions «d'element» et de «territoire» phytogeographiques. Arch. Sci. Phys. Nat. 1: 497-512.

Braun-Blanquet J. 1923. L'origine et le developpement des flores dans le massif central de France. L. Lhomme, Paris - Zürich. 
Bruyns P. V., KLAK C. \& HanáčEK P. 2011. Age and diversity in Old World succulent species of Euphorbia (Euphorbiaceae). Taxon 60(6): 1717-1733.

Bruyns P. V., Mapaya R. J. \& Hedderson T. 2006. A new subgeneric classification for Euphorbia (Euphorbiaceae) in southern Africa based on ITS and psbA-trnH sequence data. Taxon 55(2): 397-420.

Budantsev A. L. 1998. Conspectus of the genus Verbascum (Scrophulariaceae) of the Caucasian flora. Bot. Zhurn. 83(7): 128-139 (in Russian).

Chang C.-S., Kim H. \& Chang K. S. 2014. Provisional checklist of vascular plants for the Korean Peninsula Flora (KPF) (Version 1.0). Desingpost, Seoul. http://hosting03. snu.ac. kr/ quercus $1 /$ Checklist version $\% 20$ revision $\% 20$ may.pdf.

Christ H. 1867. Ueber die Verbreitung der Pflanzen in der alpinen Region der europaeischen Alpenkette. Neue Denkschr. Schw. Nat. Ges. 22: 1-84.

Davis P. H. (ed.) 1965. Flora of Turkey and East Aegean Islands. 1. University Press, Edinburgh.

Davis P. H. (ed.) 1965-1988. Flora of Turkey and East Aegean Islands. 1-10. University Press, Edinburgh.

Dorsey B. L., Haevermans T., Aubriot X., Morawetz J. J., Riina R., Steinmann V. W. \& Berry P. E. 2013. Phylogenetics, morphological evolution, and classification of Euphorbia subgenus Euphorbia. Taxon 62(2): 291-315.

EIG A. 1931. Les éléments et les groupes phytogéographiques auxiliaires dans la flore palestinienne 1. Texte. Feddes Repert. Beih. 63: 1-201.

ENGLER A. 1879. Versuch einer Entwicklungsgeschichte der extratropischen Florengebiete der nördlichen Hemisphäre. Teil 1. Die extratropischen Florengebiete der nördlichen Hemisphäre. Verlag von Wilhelm Engelmann, Leipzig.

Geltman D. V. 1996. Taxonomic notes on species of subsection Esulae of the genus Euphorbia (Euphorbiaceae) from Eastern Europe. Bot. Zhurn. 81(9): 73-89 (in Russian with English summary).

Geltman D. V. 1998. Taxonomic notes on Euphorbia esula (Euphorbiaceae) with special reference to its occurrence in the east part of the Baltic region. Ann. Bot. Fennici 35(2): 113-117.

Geltman D. V. 2009. On the taxonomy of the Euphorbia illirica (Euphorbiaceae) affinity complex. Bot. Zhurn. 94(7): 921-937 (in Russian with English summary).

Good R. 1964. The geography of the flowering plants. 3 ed. Longmans, London \& Colchester.

Govaerts R., Fernández Casas F. J., Barker C., Carter S., Davies S., Esser H.-J., Gilbert M., Hoffmann P., RaDCliffe-Smith A., Steinmann V. W., van Welzen P. C. \& Whitmore T. 2015. World Checklist of Euphorbiaceae. Facilitated by the Royal Botanic Gardens, Kew. Published on the Internet. [July 2015]. http://apps.kew.org/wcsp/.
HaYeK A. 1926. Allgemeine Pflanzengeographie. Gebrüder Borntraeger, Berlin.

Horn J. W., van Ee B. W., Morawetz J. J., Rinna R., SteinMANN V. W., Berry P. E. \& Wurdack K. J. 2012. Phylogenetics and the evolution of major structural characters in the giant genus Euphorbia L. (Euphorbiaceae). Molec. Phylogenet. Evol. 63(2): 305-326.

Horn W. H., Zhenxiang X., RiIna R., Peirson J. A., Yang Y., Dorsey B. L., Berry P. E., Davis C. C. \& Wurdack K. J. 2014. Evolutionary bursts in Euphorbia (Euphorbiaceae) are linked with photosynthetic pathway. Evolution 68(12): 3485-3504.

KAMELIN R. V. 2010. Oreocryophytic elements of the montane Middle Asian flora. Bot. Zhurn. 95(6): 730-757 (in Russian with English summary).

Kamelin R. V. 2012. Floristic division of the world land: new solutions. Bot. Zhurn. 97(12): 1481-1488 (in Russian with English summary).

Kurosawa T. 2006. The family Euphorbiaceae s.l. in Nepal. A checklist and taxonomic notes. In: H. OHBA (ed.), Himalayan Plants. 4. Bull. Univ. Mus. Univ. Tokyo 42: 155-346.

MA J. S., LIU Y. \& WeI F. N. 2013. A new species of Euphorbia (Euphorbiaceae) from Guangxi, China. Phytotaxa 87(3): 45-49.

Menitsky Y. L. 1986. Conspectus specierum generis Thymus L. (Lamiaceae) in flora Caucasi. Novosti Sist. Vyssh. Rast. 23: 117-142 (in Russian).

MeNitsky Y. L. 1992. Conspectus of Lamiaceae (Labiatae) of the Caucasian flora. Bot. Zhurn. 77(6): 63-78 (in Russian).

Pahlevani A. H. \& RiIna R. 2014. Synopsis of Euphorbia subgen. Esula sect. Helioscopia (Euphorbiaceae) in Iran with the description of Euphorbia mazandaranica sp. nov. Nordic J. Bot. 32: 257-278.

PARK K.-R. \& JANSEN R. K. 2007. A phylogeny of Euphorbieae subtribe Euphorbiinae (Euphorbiaceae) based on molecular data. J. Pl. Biol. 50(6): 644-649.

Peirson J. A., Bruyns P. V., Ritna R., Morawetz J. J. \& Berry P. E. 2013. A molecular phylogeny and classification of the largely succulent and mainly African Euphorbia subg. Athymalus (Euphorbiaceae). Taxon 62(6): 1178-1199.

Peirson J. A., Riina R., Mayfield M. H., Ferguson C. J., Urbatsch L. E., Berry P. E. 2014. Phylogenetics and taxonomy of the New World leafy spurges, Euphorbia section Tithymalus (Euphorbiaceae). Bot. J. Linn. Soc. 175: 191-228.

Pornetier N. N. 2000a. Methodical aspects of the elaboration of geographical elements in Caucasian flora. Bot. Zhurn. 85(6): 76-84 (in Russian with English summary).

Portenier N. N. 2000b. System of geographical elements of the Caucasian flora. Bot. Zhurn. 85(9): 26-33 (in Russian with English summary).

RiInA R. \& Berry P. E. (coord.) 2015. Euphorbia Planetary 
Biodiversity Inventory database. [2015]. http://www.tolkin. org/projects/72.

Riina R., Peirson J. A., Geltman D. V., Molero J., Frajman B., Pahlevani A., Barres L., Morawetz J. J., Salmaki Y., Zarre S., Kryukov A., Bruyns P. V. \& Berry P. E. 2013. A worldwide molecular phylogeny and classification of the leafy spurges, Euphorbia subgenus Esula (Euphorbiaceae). Taxon 62(2): 316-342.

Saeidi Mehrvarz S. \& Ghayormand M. 2015. Euphorbia khorasanica (Euphorbiaceae), a new species from Iran. Ann. Bot. Fennici 52(1-2): 38-40.

Skvortsov A. K. 1966. Obzor iv Kavkaza i Maloy Azii. Trudy Bot. Inst. Akad. Nauk Armyansk. S.S.R. 15: 91-141.

Steinmann V. W. \& Porter J. M. 2002. Phylogenetic relationships in Euphorbieae (Euphorbiaceae) based on ITS and $n d h \mathrm{~F}$ sequence data. Ann. Missouri Bot. Gard. 89(4): 453-490.

TAKHTAJAN A. 1986. Floristic regions of the world. University of California Press, Berkeley \& Los Angeles.
Tolmachev A. I. 1974. Vvedenie v geografiyu rasteniy. Izdatel'stvo Leningradskogo Universiteta, Leningrad.

WU Z. \& WU S. 1998. A proposal for a new floristic kingdom (realm) - the E. Asiatic Kingdom, its delimitation and characteristics. In: A. L. Zhang \& S. G. Wu (eds), Floristic characteristics and diversity of East Asian plants, pp. 3-42. China Higher Education Press, Beijing \& SpringerVerlag, Berlin.

Yang Y., RiIna R., Morawetz J. J., Haevermans T., Aubriot X. \& Berry P. E. 2012. Molecular phylogenetics and classification of Euphorbia subgenus Chamaesyce (Euphorbiaceae). Taxon 61(4): 764-789.

Yurtsev B. A. \& KAMELIN R. V. 1991. Osnovnye ponyatiya $i$ terminy floristiki: Uchebnoe posobie po spetskursu. Izdatel'stvo Permskogo Universiteta, Perm.

Zimmermann N. F. A., Ritz C. M. \& Hellwig F. H. 2010. Further support for the phylogenetic relationships within Euphorbia L. (Euphorbiaceae) from nrITS and trnL-trnF IGS sequence data. Pl. Syst. Evol. 286: 39-58. 